\title{
Estimating site-specific spawning parameters for a spawning aggregation: an example with spotted seatrout
}

\author{
Katherine Zarada $^{1, *}$, Sarah Walters Burnsed ${ }^{2}$, Joel Bickford ${ }^{2}$, \\ Nicholas Ducharme-Barth ${ }^{1}$, Robert N. M. Ahrens ${ }^{1}$, Susan Lowerre-Barbieri ${ }^{1,2}$ \\ ${ }^{1}$ School of Forestry Resources and Conservation, Department of Fisheries and Aquatic Sciences, University of Florida, \\ Gainesville, FL 32611, USA \\ ${ }^{2}$ Florida Fish and Wildlife, Fish and Wildlife Research Institute, St. Petersburg, FL 33701, USA
}

\begin{abstract}
An understanding of individual variability in spatio-temporal spawning behavior is important to understand productivity and inform stock assessments and management actions. Spotted seatrout Cynoscion nebulosus, popular both with recreational anglers and researchers due to their accessibility and catchability, have frequently been the subject of reproductive studies at the population level. We used passive acoustic telemetry to estimate spotted seatrout spawning interval and period and investigate spawning behaviors at the individual level. A total of 43 spotted seatrout (27 females, 16 males) were acoustically tagged and tracked over 3 consecutive spawning seasons with a high-density array deployed at a residential spawning aggregation site in Tampa Bay, Florida. The probability of a fish being at the spawning site and instantaneous loss from the population $(Z)$ was estimated using a Bayesian framework-Cormack Jolly Seber model. Most fish exhibited intra-seasonal site fidelity, with males and larger fish of both sexes exhibiting a greater probability of being at the spawning site. A similar pattern with total length and sex was seen in $Z$. There were sex-dependent differences in movement within the array, with females traveling a larger distance between single detections while males had a higher total distance traveled within the array. This study demonstrates how acoustic telemetry can be combined with Bayesian models to estimate sex- and length-dependent spawning parameters for an aggregating marine species that exhibits temporal migration to and from a spawning site.
\end{abstract}

KEY WORDS: Reproductive potential $\cdot$ Spawning frequency $\cdot$ Reproductive behavior

\section{INTRODUCTION}

Species persistence is dependent on reproductive success, which is the ability of an adult to produce offspring that survive to reproduce (Stearns 1992). However, due to the inability to directly observe fish spawning, and the weak correlation between reproductive potential measures and recruitment, reproductive success in fisheries can be difficult to quantify. In fisheries assessments, the relationship between reproductive potential and resulting recruitment has

\footnotetext{
${ }^{*}$ Corresponding author: kzarada@ufl.edu
}

traditionally been modeled through the stock-recruit relationship, with spawning stock biomass (SSB) used as an indicator of reproductive potential related to the resulting offspring recruiting to the fishery (Ricker 1954, Beverton \& Holt 1957). Under the simplifying assumption of a relatively stable age distribution, total egg production (TEP) is assumed to be proportional to female SSB and a relative measure of fecundity per unit biomass (Beverton \& Holt 1957, Marshall et al. 1998). However, SSB is often a poor predictor of egg production (Rothschild \& Fogarty 1989, Trippel 1999,

() The authors 2019. Open Access under Creative Commons by Attribution Licence. Use, distribution and reproduction are unrestricted. Authors and original publication must be credited. 
Marshall et al. 2003) due to a range of age- and sizedependent effects including maturation schedules and sex-specific behaviors affecting sex ratios, increased relative fecundity and reproductive success with age and size of the spawners (Lambert 1987, Secor 2000, Wright \& Trippel 2009).

Quantifying size- and sex-specific spawning frequency, batch fecundity, duration of individual spawning periods, and the number of individuals spawning - parameters needed to estimate TEP — is particularly challenging when attempting to understand behavior in batch spawners with indeterminate fecundity. A spawning aggregation can occur at a site over the duration of the population's spawning season, but because individuals spawn on different dates (Lowerre-Barbieri et al. 2013), traditional capture-based methods may not be able to accurately sample fish to estimate spawning frequency (Hunter \& Macewicz 1985, Lowerre-Barbieri et al. 2016). Fundamental assumptions of these methods are often violated as individuals that participate in spawning aggregations move between spawning and feeding grounds (temporal migration) throughout the spawning period. Since many marine fish participate in spawning aggregations (Russell et al. 2012) and are indeterminate spawners that spawn multiple times over a protracted spawning season (Murua \& Saborido-Rey 2003), it is important to incorporate movement when estimating spawning parameters.

Recent advances in acoustic telemetry make it possible to track fine-scale movements of fish over time, and to estimate length-dependent effects on spawning behaviors (Robichaud \& Rose 2003, Semmens et al. 2010, Crossin et al. 2017). This capacity to track an individual over space and time gives insights into behaviors and patterns that traditional capture-based methods are not capable of detecting. We evaluated individual spawning site selection of spotted seatrout Cynoscion nebulosus over 3 spawning seasons through the use of acoustic telemetry at a fisheries spawning aggregation site (FSA). Specifically, we assessed (1) the area from which individuals are drawn to this FSA and whether this varies with sex; (2) interseasonal and inter-annual spawning site fidelity; and (3) the number of times an individual moves to this site to spawn within a given spawning season. Since telemetry studies are often limited by small sample sizes, we employed a Bayesian model to estimate spawning parameters. With careful prior consideration, Bayesian models are able to handle small sample sizes better than frequentist methods (Dorazio 2016). The Bayesian framework-Cormack Jolly Seber (CJS) mark-recapture model estimated the probabil- ity of being at the spawning site and instantaneous loss from the population $(Z)$. We conclude by discussing limitations of traditional capture-based methods to estimate annual fecundity in indeterminate species versus our proposed space-based method.

\section{MATERIALS AND METHODS}

\subsection{Ethics}

No specific permission for sampling was required, as sampling was conducted by the Florida Fish and Wildlife Conservation Commission's Fish and Wildlife Research Institute. However, every effort was made to meet all ethical standards and no protected species were sampled.

\subsection{Study site}

This study was conducted at Bunces Pass, an inlet at the mouth of Tampa Bay, Florida over a $3 \mathrm{yr}$ period from 2007-2009. Bunces Pass is approximately $300 \mathrm{~m}$ across and has a maximum depth of $8.5 \mathrm{~m}$. The bottom is primarily sand and shell hash with no submerged aquatic vegetation or oyster reefs. The inlet is tidally driven, and the current can reach a maximum of $1 \mathrm{~m} \mathrm{~s}^{-1}$ (Lowerre-Barbieri et al. 2009). Although Walters et al. (2009) found a widespread distribution of spawning activity throughout Tampa Bay, with aggregation sites predominantly in shallow waters near seagrass, Bunces Pass was found to be the most consistently used area, as indicated by daily and seasonal aggregation level sound, even though it does not have similar features to other spawning sites (Lowerre-Barbieri et al. 2009, Walters et al. 2009). Furthermore, the spawning fraction (determined through the percentage of females with hydrated or mature oocytes) at the inlet was $97 \%$ (Lowerre-Barbieri et al. 2009).

\subsection{Fish tagging}

Spotted seatrout were captured during spawning season via seine net $(122 \times 2.4 \mathrm{~m}$ knotless haul seine, $0.6 \mathrm{~cm}$ delta mesh, with a center bag measuring $2.4 \times$ $2.4 \times 2.4 \mathrm{~m})$ in the early evening $(17: 35-21: 44 \mathrm{~h})$ from 25 April to 31 May 2007 and from 5 May to 15 May 2008 (Table 1). All tagged fish were captured, tagged, and released in the Bunces Pass spawning site, and confirmed to be in spawning condition by 
Table 1. Tag number, sex, total length (TL), total period detected (TP), days detected (DD) spawning interval (SI), implant date, and tag type for each spotted seatrout male (M) or female (F) detected in the spawning site

\begin{tabular}{|c|c|c|c|c|c|c|c|}
\hline Tag & Sex & $\begin{array}{c}\mathrm{TL} \\
(\mathrm{mm})\end{array}$ & $\begin{array}{l}\text { TP } \\
\text { (d) }\end{array}$ & $\begin{array}{l}\text { DD } \\
\text { (d) }\end{array}$ & $\begin{array}{l}\text { SI } \\
\text { (d) }\end{array}$ & $\begin{array}{l}\text { Implant date } \\
\text { (dd/mm/yy) }\end{array}$ & $\begin{array}{l}\text { Tag } \\
\text { type }\end{array}$ \\
\hline 1 & $\mathrm{~F}$ & 349 & 42 & 13 & 3.50 & $25 / 04 / 07$ & V9 \\
\hline 3 & M & 355 & 66 & 5 & 16.5 & $30 / 04 / 07$ & V9 \\
\hline 5 & M & 388 & 40 & 3 & 20 & $01 / 05 / 07$ & V9 \\
\hline 6 & $\mathrm{~F}$ & 438 & 44 & 6 & 8.80 & 03/05/07 & V9 \\
\hline 8 & M & 362 & 13 & 9 & 1.62 & $16 / 05 / 07$ & V9 \\
\hline 9 & M & 371 & 14 & 5 & 3.50 & $16 / 05 / 07$ & V9 \\
\hline 10 & $\mathrm{~F}$ & 334 & 4 & 2 & 4 & $16 / 05 / 07$ & V9 \\
\hline 13 & M & 425 & 30 & 7 & 5 & $25 / 04 / 07$ & V9 \\
\hline 15 & $\mathrm{~F}$ & 494 & 1 & 2 & 1 & $01 / 05 / 07$ & V9 \\
\hline 17 & F & 516 & 85 & 11 & 8.50 & $01 / 05 / 07$ & V9 \\
\hline 19 & $\mathrm{~F}$ & 463 & 89 & 4 & 29.67 & $01 / 05 / 07$ & V13 \\
\hline 20 & M & 500 & 94 & 47 & 2.04 & $01 / 05 / 07$ & V13 \\
\hline 21 & $\mathrm{~F}$ & 601 & 41 & 4 & 13.67 & 01/05/07 & V13 \\
\hline 22 & $\mathrm{~F}$ & 494 & 90 & 14 & 6.92 & $01 / 05 / 07$ & V13 \\
\hline 23 & F & 482 & 16 & 6 & 3.20 & $01 / 05 / 07$ & V13 \\
\hline 25 & F & 480 & 64 & 16 & 4.27 & $16 / 05 / 07$ & V13 \\
\hline 28 & $\mathrm{~F}$ & 571 & 54 & 13 & 4.50 & $22 / 05 / 07$ & V13 \\
\hline 32 & M & 420 & 1 & 2 & 1 & $31 / 05 / 07$ & V13 \\
\hline 33 & $\mathrm{~F}$ & 472 & 36 & 9 & 4.50 & $31 / 05 / 07$ & V13 \\
\hline 34 & M & 430 & 17 & 10 & 1.89 & 05/05/08 & V13 \\
\hline 35 & $\mathrm{~F}$ & 528 & 65 & 8 & 9.29 & 05/05/08 & V13 \\
\hline 36 & F & 510 & 75 & 10 & 8.33 & 05/05/08 & V13 \\
\hline 37 & F & 428 & 78.5 & 10 & 9.03 & 05/05/08 & V13 \\
\hline 39 & $\mathrm{~F}$ & 464 & 11 & 2 & 11 & $14 / 05 / 08$ & V13 \\
\hline 40 & $\mathrm{~F}$ & 465 & 79.5 & 11.5 & 9.46 & $14 / 05 / 08$ & V13 \\
\hline 41 & M & 390 & 25 & 4 & 8.33 & $15 / 05 / 08$ & V13 \\
\hline 42 & M & 440 & 115 & 56.5 & 2.27 & $15 / 05 / 08$ & V13 \\
\hline 44 & M & 508 & 52 & 4.5 & 20.6 & $15 / 05 / 08$ & V13 \\
\hline 45 & M & 435 & 7 & 2.5 & 4.75 & $15 / 05 / 08$ & V13 \\
\hline 46 & M & 486 & 94.5 & 9 & 11.22 & $15 / 05 / 08$ & V13 \\
\hline
\end{tabular}

observing flowing milt or eggs when pressure was applied to the abdomen. Lowerre-Barbieri et al. (2013) demonstrated that the described surgical procedure and tagging process does not inhibit spawning or gonadal development in spotted seatrout. Prior to the surgery, fish were stored in a flow-through 7951 live well. The surgical procedure followed Lowerre-Barbieri et al. (2013), but due to regulatory changes, anesthesia was not administered. Surgeries lasted approximately $7 \mathrm{~min}$, during which a coded Vemco tag was intra-peritoneally implanted and the incision site closed with a dissolvable suture. Fish were tagged with either V9 (146 dB output, $149 \mathrm{~d}$ battery life, 15-45 d random inter-pulse delay) or V13 (147 dB output, 540 d battery life, 30-90 s random inter-pulse delay) Vemco coded transmitters. Spotted seatrout spawning seasons range from April to September (Brown-Peterson et al. 2001). To extend battery life and collect data over multiple spawning seasons, V13 transmitters were coded to turn off outside of the spawning season (21 September21 March). In 2007, 30 individuals were tagged; 10 (5 females, 5 males) were tagged with V9 tags that were active for the one spawning season in which they were tagged. The other 20 (16 females, 4 males) were tagged with V13 tags that were active for 3 spawning seasons. In 2008, 13 additional fish (6 females, 7 males) were tagged with V13 tags (Table 1). Two of the 2008 V13 tags (tag numbers 40 and 42) were programmed to remain on outside of the spawning season for other research. Because our research was focused on the spawning population, spatiotemporal behaviors outside of the spawning season by these 2 tags were not included.

\subsection{Receiver array}

An array of 42 acoustic receivers (VR2, Vemco) was deployed to monitor the Bunces Pass inlet and surrounding areas (Fig. 1). The receiver array is an extension of that used in the 2005 study (LowerreBarbieri et al. 2013), and the same as described in Lowerre-Barbieri et al. (2014). To simplify spatial interpretations, the array was separated into 7 zones (Lowerre-Barbieri et al. 2014) (Fig. 1). A core array of 17 receivers with overlapping ranges was deployed in the Bunces spawning site (zones 2 and 3). Receiver array coverage of the spawning site was determined by identifying the area of aggregation-level courtship sounds and the presence of actively spawning females (Walters et al. 2009, Lowerre-Barbieri et al. 2009). Previous long-term testing at the spawning site demonstrated that a range of $85 \mathrm{~m}$ was the maximum range for consistent detections (Lowerre-Barbieri et al. 2013). Therefore, receivers in the spawning site were placed within an $85 \mathrm{~m}$ range of each other to ensure complete coverage and create an acoustic positioning system. Telemetry arrays with overlapping coverage (acoustic positioning systems) are able to triangulate individual positions with high accuracy ( 1-2 m) (Espinoza et al. 2011). Although the receivers were not VPS (Vemco position system), control tags were used to estimate position error. The 3 control tags (69 kHZ Vemco V9sc-2L 139 dB with a $60 \mathrm{~s}$ fixed delay) were deployed in zones 2, 5, and 6 . The movements to and from the spawning grounds occurred along the east and west axis, and therefore our estimation of error focused on this axis. The mean easting position error of the control tags was $35.1 \pm$ $0.69 \mathrm{~m}$. Errors $>200 \mathrm{~m}$ only occurred $0.1 \%$ of the time ( $\mathrm{n}=2$ out of 1920 ) and the tags had a detection rate of $99 \%$ (Lowerre-Barbieri et al. 2014). 


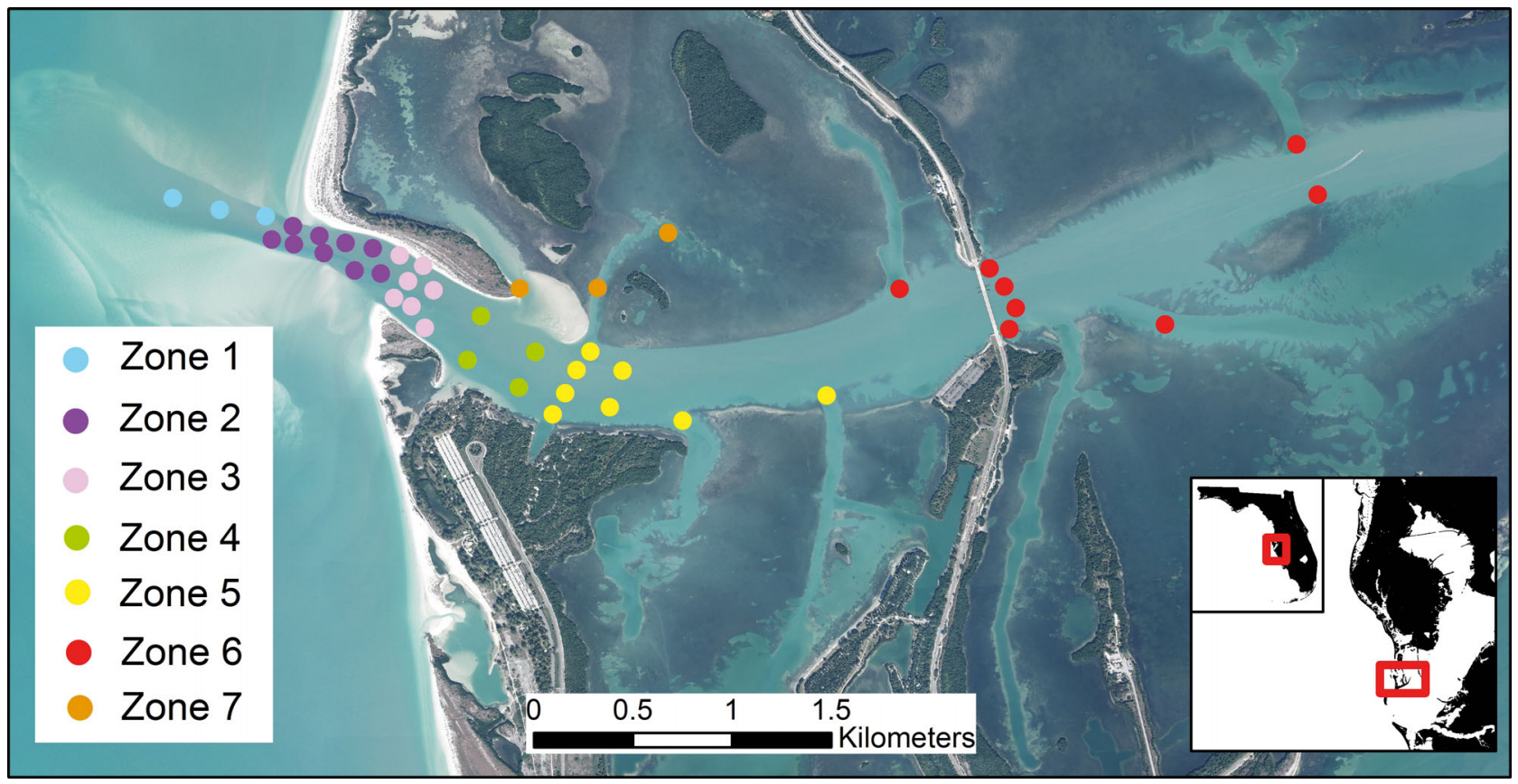

Fig. 1. Receiver array for spotted seatrout in Bunces Pass in Tampa Bay with zone assignments by color. Zones 2 and 3 delineate the core of 17 receivers and encompasses the spawning site; zone 1 consists of 3 receivers moored west of the spawning site/core array in the Gulf of Mexico; zones 4, 5, 6, and 7 are composed of 23 receivers in different areas of the estuary east of the spawning site/core array

\subsection{Data analysis}

The center of activity of each fish was estimated using the weighted means methods described in Simpfendorfer et al. (2002) for 10 min time bins. This time step was chosen to create data that was finescale enough to detect movement patterns while ensuring enough detections per bin to get accurate location estimations. The average number of detections was $16.14 \pm 11.39 \mathrm{bin}^{-1}$. Spurious detections $(\mathrm{n}=21)$ were defined as a single detection from a transmitter code (fish tag) in the zones with complete acoustic coverage ( 2 and 3 ) during a $24 \mathrm{~h}$ period and were filtered from the data set. The best $24 \mathrm{~h}$ cycle to group detections (Douglas et al. 2009) was determined by Lowerre-Barbieri et al. (2013) through monitoring hourly fish presence and behavioral patterns in the spawning site (zone 2 or 3 ). Since the fewest fish were detected in the spawning site from 06:00-06:59 h, a $24 \mathrm{~h}$ cycle from 07:00-06:59 h was adopted for this study. The zones were separated into the spawning site (zone 2 and 3 ) and surrounding areas (zones 1,4-7). For the spawning site, the total detection period (TP), days detected (DD), and days between detections were calculated for all fish for each spawning season. TP was defined as the num- ber of days between the first and last detection and $\mathrm{DD}$ as the number of days fish were detected.

\subsubsection{Spawning site fidelity}

Intra-seasonal FSA site fidelity was assessed based on DD and TP for individuals. Inter-annual site fidelity was determined by the presence of detections in the FSA over multiple years during the $3 \mathrm{yr}$ study period.

\subsubsection{Movement patterns}

Individual movements within the array were examined using the $\mathrm{R}$ package 'adehabitatLT' (Calenge 2006). Total and maximum distance traveled within the array were calculated for all individuals during the 2007 spawning season using the function 'as.ltraj'. Total distance within the array was the sum of all the distances between detections in the array, while maximum distance was the largest distance between 2 consecutive relocations. A linear model was used to examine the effects of sex and total length (TL) on the maximum distance traveled. Max- 
imum and total distances traveled within the array for males and females were compared with $t$-tests.

\subsubsection{Spawning interval and apparent loss}

Spawning interval was estimated for all individuals during all 3 spawning seasons as the number of days between detections at the FSA. At the population level, spawning interval was calculated as the reciprocal of the probability of being in the spawning grounds (equivalent to estimating spawning interval from the reciprocal of the spawning fraction; Hunter \& Macewicz 1985), which was estimated for sex and sex-TL groups using a hierarchical CJS model. The CJS model was modified to be hierarchical from Kéry \& Schaub (2012).

Three TL groups were used in the model: small (<400 mm TL), medium (400-500 mm TL), and large (<500 mm TL). TL groups were chosen to correspond roughly to the Florida recreational catch slot size for spotted seatrout. Anglers are allowed to keep 4 fish $\mathrm{d}^{-1}$ between 381 and $508 \mathrm{~mm} \mathrm{TL}$, and one of those can be $>508 \mathrm{~mm}$ TL (Murphy et al. 2011). The TL groups result in medium and large fish being vulnerable to the fishery while small fish are not. Sample sizes by TL groups differed for both sexes (Table 2).

The model data was a matrix of presence and absence detections of individuals in the spawning site (zones 2 and 3, where there is complete coverage) during the temporal window associated with spawning, as defined from presence of aggregation

Table 2. Probability of spotted seatrout being in the spawning site, determined from the Cormack Jolly Seber Bayesian model. Groupings were by sex and sex-total length bins. Males had the highest probability of being in the spawning site, indicating a higher spawning frequency. Out of the total length bins, the largest females had the highest probability, demonstrating that the largest females have a higher spawning frequency than smaller females

\begin{tabular}{|lcccc|}
\hline Grouping & Mean & CI & Days & $\mathrm{n}$ \\
\hline Female & 0.126 & $0.037-0.368$ & 6.76 & 17 \\
Male & 0.327 & $0.037-0.863$ & 2.65 & 14 \\
Female_small & 0.135 & $0.086-0.211$ & 6.90 & 2 \\
Female_medium & 0.105 & $0.083-0.129$ & 9.43 & 10 \\
Female_large & 0.144 & $0.108-0.189$ & 6.76 & 5 \\
Male_small & 0.160 & $0.109-0.223$ & 6.06 & 5 \\
Male_medium & 0.428 & $0.366-0.491$ & 2.34 & 7 \\
Male_large & 0.445 & $0.357-0.536$ & 2.24 & 2 \\
\hline
\end{tabular}

level sound (17:00-06:00 $\mathrm{h}$; Lowerre-Barbieri et al. 2009). The CJS model only used data from the first season of detection for each individual. Second season information was removed for individuals that exhibited inter-annual site fidelity to ensure that survival was daily, and to prevent bias on the probability of entering the FSA for individuals that survived and returned the following season.

Conventional state-space models are composed of an observation and state process, where the observation process is the probability of recapturing an individual and the state process is the probability of survival. In this study, the recapture process was detecting an individual through telemetry at the FSA. Since we assumed complete detection of individuals within zones 2 and 3, instead of an observation process, we modeled the probability of an individual being at the FSA $(p)$. The state process was daily survival $(\varphi)$. Probability of being at the FSA by TL group $\left(p_{g}\right)$ was drawn from a sex-specific hyper-distribution with a sex-specific mean probability of being at the FSA $\left(\mu_{\rho, s}\right)$ and a sex-specific precision $\left(\tau_{\rho_{s} s}\right)$ :

$$
p_{g} \sim \operatorname{Normal}\left(\mu_{\rho, s}, \tau_{\rho, s}\right)
$$

The probability of survival by TL group $\left(\varphi_{g}\right)$ was drawn from sex-specific hyper-distributions with sex-specific mean probability of survival $\left(\mu_{\varphi, s}\right)$ and a sex-specific precision $\left(\tau_{\varphi, s}\right)$ :

$$
\varphi_{g} \sim \operatorname{Normal}\left(\mu_{\varphi, s}, \tau_{\varphi, s}\right)
$$

A time component was added as a power function to survival to account for the days between overall detections and seasons. The time component ensured survival stayed daily and constant.

Sex-specific means $\left(\mu_{\varphi, s} ; \mu_{\rho, s}\right)$ were drawn from uniform distributions from 0-1 and standard deviations were drawn from uniform distributions from 0-5. Precision $\left(\tau_{\varphi, s i} \tau_{\rho, s}\right)$ was calculated as the inverse of the variance. The probabilities were bound between 0 and 1 using an inverse logit transformation.

Posterior distributions were estimated for each sex and TL grouping using the Markov chain Monte Carlo (MCMC) method implemented in JAGS using the package 'rjags' (Plummer 2016) in the program R (R Core Team 2017). Four chains were used for each parameter estimation, with a burn in of 10000 and a sample of 50000 . Posterior distributions for the probability of being in the spawning grounds and the instantaneous population loss were estimated by sex as well as by sex and TL groups. Chain convergence was visually tested and was confirmed with the Gelman and Rubin multiple sequence diagnostic, Geweke diagnostic, and Hei- 
delbery and Welch convergence diagnostic from the R package 'coda' (Plummer et al. 2006). The spawning interval (in days) was calculated as the reciprocal of the mean of the probability of being at the FSA. The daily loss posterior distribution was extrapolated to yearly $Z$. The population was open; therefore, loss is not strictly mortality but instead encompasses emigration, fishing mortality, and natural mortality. Code for the CJS model is available on GitHub at kzarada/SpottedSeatrout.

\subsubsection{Spawning period}

Spawning period was modeled using a generalized linear model (GLM) with a negative binomial distribution using the data from all individuals from all 3 seasons. TP is equivalent to the spawning period. Individuals with a total period $<2 \mathrm{~d}$ were removed from the model to remove the bias of individuals who may have died or did not return to the spawning grounds. The fixed effects were TL and year. Model dispersion was checked using the function 'P_disp' from the 'msme' package (Hilbe \& Robinson 2018).

\section{RESULTS}

Spotted seatrout were tagged at the beginning of both the 2007 ( $\mathrm{n}=30$ ) and 2008 ( $\mathrm{n}=13$ ) spawning seasons, for a total of 43 individuals. Female $(n=27)$ lengths ranged from 334-601 mm TL with a mean of $470.5 \mathrm{~mm}$. Male $(\mathrm{n}=16)$ lengths ranged from 355$510 \mathrm{~mm}$ TL with a mean of $426.8 \mathrm{~mm}$. Fish were tracked over the course of 3 spawning seasons for a total of 92500 detections in 2007, 88438 detections in 2008, and 84941 detections in 2009.

\subsection{Spawning site fidelity}

Most individuals exhibited intra-seasonal site fidelity ( $\mathrm{n}=31,72 \%$ ), returning to the FSA multiple times during the spawning season. Of the 43 tagged individuals, 11 did not re-enter the spawning site post-tagging (10 females, 1 male). Most of these fish were detected on only a few dates (6 or less) relatively soon after being tagged. However, tags 24 and 26 were detected on 25 and 32 d respectively. Two fish (tags 2 and 32) were only detected at the FSA one time post-tagging (Fig. 2). Note that tag 12

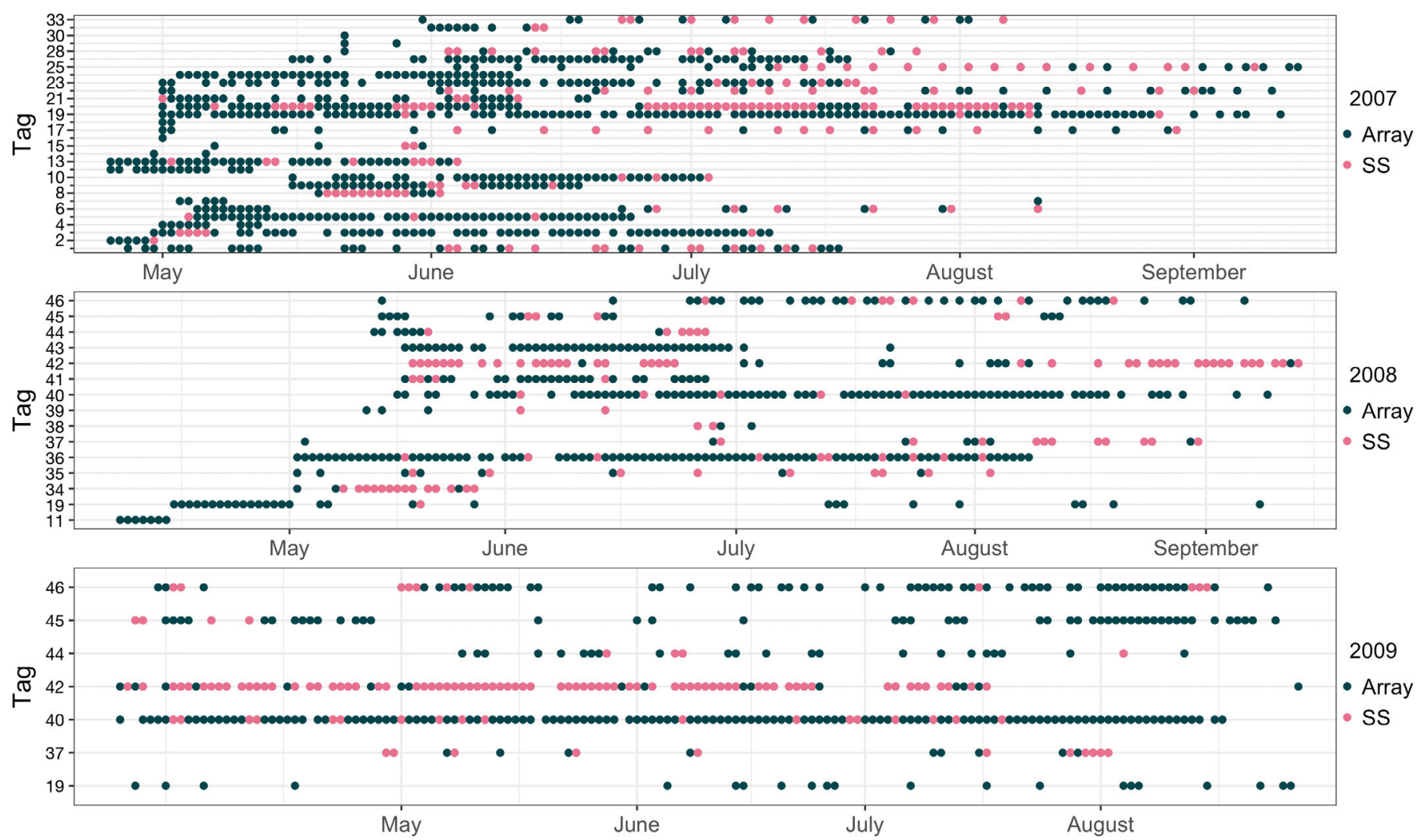

Fig. 2. Daily detections of spotted seatrout at the spawning site (SS; zones 2 and 3) and surrounding areas (Array; zones 1 and $4-7$ ) over the 3 spawning seasons monitored. Top panel has tick marks on $y$-axis labelled for every other tag. Note that tags 12 , 27 and 31 do not exist 


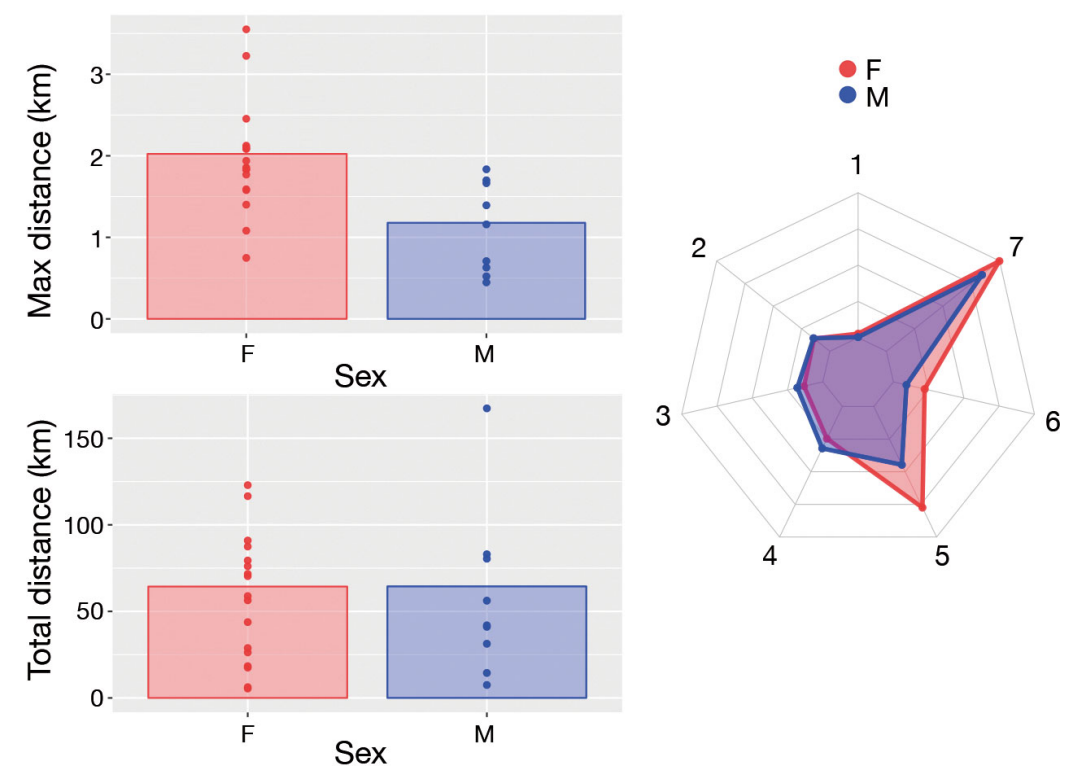

Fig. 3. Maximum (top) and total (bottom) distance traveled by male and female spotted seatrout in 2007. Bars: mean distance; dots: individual distances. Radar plot (right) demonstrates the differences in zone usage by males and females for the 2007 spawning season; most detections occurred in zone 7 . Females were detected more often than males in zones 5, 6, and 7 while males were detected more often than females in zones 2,3 , and 4

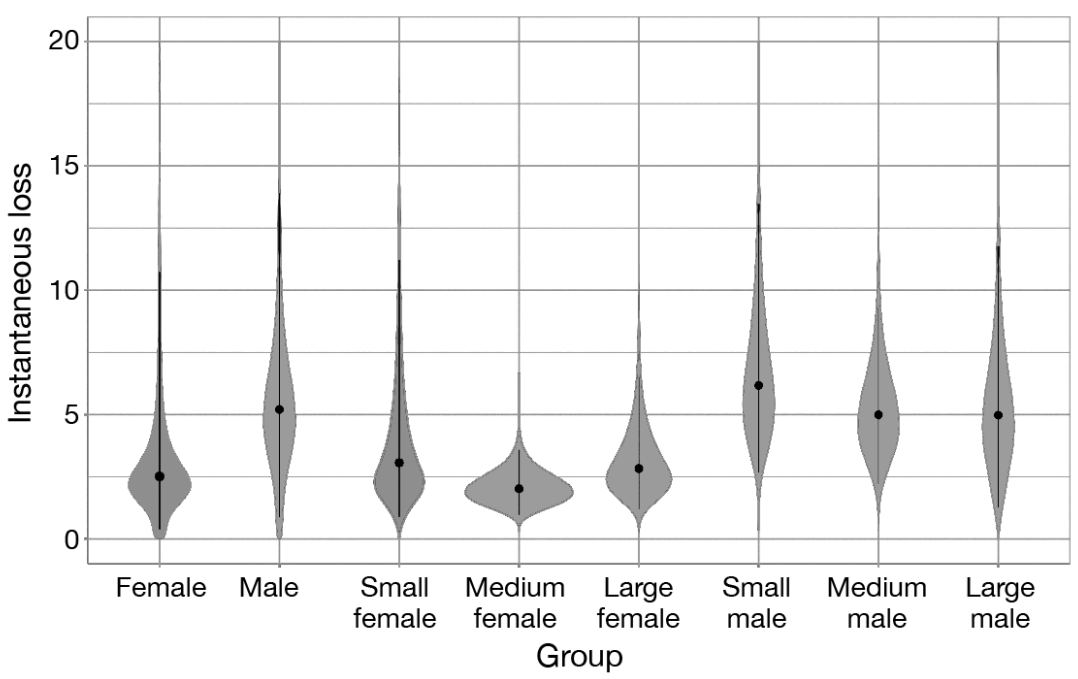

Fig. 4. Instantaneous loss from the population for the sex mean (female and male) and total length bins of small ( $<400 \mathrm{~mm}$ total length, TL), medium (400$500 \mathrm{~mm}$ TL), and large (>500 mm TL) male and female spotted seatrout. Points: median; lines: $95 \%$ credible interval; $y$-axis limit: 20 . Males have a higher loss from the population, with medium sized males having the highest loss. The smallest females have the highest loss from the female population

was again detected in 2009 but not at the FSA site. Inter-annual site fidelity was greater for fish tagged in 2008 ( $\mathrm{n}=$ 13), with 6 of these fish ( 2 females, 4 males) again detected in 2009 and all detected within the FSA site (Fig. 2).

\subsection{Movement patterns}

Total distance traveled by individuals within the array during the 2007 spawning season ranged from 5.41-167.28 km with a mean of $57.7 \mathrm{~km}$ and a standard deviation of 40.1 (Fig. 3). Out of the 43 tagged fish, 22 females $(81.5 \%)$ and 8 males $(50 \%)$ were detected in the eastern-most part of the array, zone 6 (Fig. 3). Zone 7 had the most detections in 2007 ( $n=37$ 157) (Fig. 3).

Females traveled greater distances than males. The linear model showed a significant negative correlation between being male and the maximum distance traveled within the array $(\beta=$ $-0.10, \mathrm{SE}=0.37, \mathrm{p}>0.05)$. There was no relationship between maximum distance and TL or between total distance traveled and sex and TL. There was a significant difference in the means between the maximum distance traveled by females and males ( $p>0.05)$. Females had a mean maximum distance of $2.02 \mathrm{~km}$ with a standard deviation of 0.68 while males had a mean maximum distance of $1.19 \mathrm{~km}$ with a standard deviation of 0.56 (Fig. 3).

\subsection{Apparent loss}

The posterior probabilities for the yearly $Z$ for females had a median of 2.55 and a credible interval of 0.41119.207 while the probability of loss for males had a median of 5.315 and a credible interval of 0.883-27.019 (Fig. 4;

was lost post-implantation and tags 27 and 31 were not implanted. Inter-annual site fidelity was not as common. Of the 20 fish tagged with V13 tags in 2007 , only 2 fish (tags 11 and 19, both females) were relocated the following spawning season, but only one of them was detected at the FSA site (tag 19). This fish data $>20$ not shown). When the grouping was sex and TL, the smallest males had the highest loss with a median of 6.174 and a credible interval of 2.66813.588. The smallest females had the highest loss out of the females with a median of 3.066 and a credible interval of 0.866-11. (Fig. 4, Table 3). 
Table 3. Instantaneous loss from the Cormack Jolly Seber Bayesian model. Groupings were sex and sex-total length bins. Males had a higher instantaneous loss compared to females, but when the total length bins were used both the largest males and the largest females had the highest instantaneous loss from the population

\begin{tabular}{|lccc|}
\hline Grouping & Mean & CI & $\mathrm{n}$ \\
\hline Female & 2.553 & $0.411-19.207$ & 17 \\
Male & 5.315 & $0.882-27.019$ & 14 \\
Female_small & 3.066 & $0.886-11.346$ & 2 \\
Female_medium & 2.017 & $0.967-3.598$ & 10 \\
Female_large & 2.832 & $1.174-6.518$ & 5 \\
Male_small & 6.174 & $2.668-13.588$ & 7 \\
Male_medium & 4.994 & $2.199-9.275$ & 2 \\
Male_large & 4.981 & $1.265-11.845$ & 17 \\
\hline
\end{tabular}

\subsection{Spawning interval}

At the individual level, the number of DD on the spawning site for all spawning seasons ranged from 1-71 d with a mean of $11 \mathrm{~d}$, and the number of days between spawns ranged from 1-34 $\mathrm{d}$ with a mean of $8.21 \mathrm{~d}$ for both sexes.

The posterior probability from the CJS model of being in the spawning site for females had a median of 0.126 and a credible interval of $0.037-0.368$, while males had a median of 0.327 and a credible interval of $0.037-0.863$ for the 2007 and 2008 spawning seasons (Fig. 5, Table 2). These probabilities result in an average spawning interval of $7.94 \mathrm{~d}$ for females and

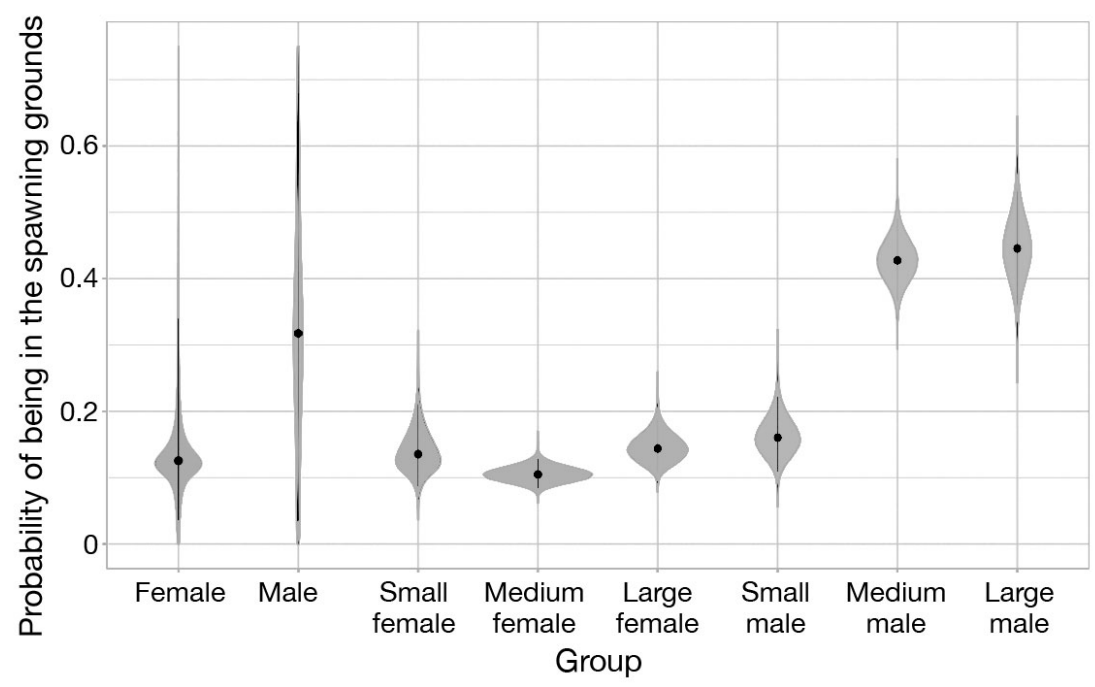

Fig. 5. Poster distribution of the probability of spotted seatrout being in the spawning site for the first season of detection for each individual. Points: median; lines: $95 \%$ credible interval. Males have a higher probability of entering the spawning site than females, and the largest males have the highest probability out of the sampled population. The largest females have the highest mean out of the female population, but the difference is not statistically significant
$3.06 \mathrm{~d}$ for males. When the grouping was sex/TL bins, the largest males had the highest probability of being at the spawning site with a median of 0.445 and a credible interval of $0.357-0.536$, which resulted in a spawning interval of $2.25 \mathrm{~d}$. Out of the females, the largest females had the highest probability of being at the spawning site, with a median of 0.144 and a credible interval of $0.108-0.189$, resulting in a spawning interval of $6.94 \mathrm{~d}$ (Fig. 5, Table 2).

\subsection{Spawning period}

The spawning period was variable, but there was a significant correlation with TL. The TP for individuals that survived tag implantation and returned to the spawning site post-tagging ( $\mathrm{TP}>2$ ) ranged from 4-136 d with a mean of $54.94 \mathrm{~d}$ and a standard deviation of 35.97. TL had a significant positive correlation with TP (TL: $\beta=0.004, p<0.02$ ), but sex was not a significant factor. For all individuals, the 2007 season lasted for $148 \mathrm{~d}$ with the first detection on 26 April, the first date fish were tagged, and the last detection on 21 September. In 2008, the population spawning season was $175 \mathrm{~d}$ with the first detection on 21 March and the last detection was 12 September. The 2009 season was $159 \mathrm{~d}$ with the first detection on 27 March and the last detection on 2 September. These dates reflect detections of individuals whose tags turned off outside of the spawning season. The 2 individuals with tags that remained on were detectable year-round. Detections of these 2 fish outside of the spawning season showed different diel periodicity than detections during the spawning season and were primarily east of the FSA site.

\section{DISCUSSION}

SSB and TEP are the measures of reproductive potential commonly used in stock assessments. For fish with indeterminate fecundity, annual fecundity is estimated as the product of batch fecundity and spawning frequency, or number of spawns season ${ }^{-1}$ (Hunter \& Macewicz 1985). Spawning frequency is equal to the number of days in the spawning season divided by the spawning interval, where the spawning interval is traditionally calculated as the reciprocal of the propor- 
tion of active spawners (Hunter \& Macewicz 1985). However, for fish that form spawning aggregations, with temporal migration from the FSA site, the traditional method of estimating spawning frequency will not be accurate due to immigration/emigration from the spawning site. In addition, there are no histological markers of active spawning in males and thus it has not been possible to estimate male spawning frequency. Acoustic telemetry makes it possible to track individuals to and from the FSA site and, when combined with the known proportion of active spawners at that site, can be used to estimate spawning frequency at a given site. The use of telemetry allows insight into spawning behaviors that impact reproductive success such as site fidelity, movement, spawning period, spawning interval, and loss from the population. These findings are discussed in greater detail for each reproductive parameter and behavior.

\subsection{Spawning site fidelity}

Spawning site selection is a balance between maximizing offspring survival (Charnov 1976, Siceloff \& Howell 2013) and food intake while minimizing predation risk (Ciannelli et al. 2015). Site fidelity creates potential for site-specific differences in reproductive productivity and resilience (Lowerre-Barbieri et al. 2017), and understanding spawning site fidelity is important for efficient management of critical habitat and for discovering complex spatial use and migration patterns (Young et al. 2016). Both intra-seasonal and inter-annual site fidelity was detected at this spawning site. Previous research has found other spotted seatrout aggregations to be composed of a mix of spawners and non-spawners (spawning fraction ranging from 7-44 \%; reviewed by Brown-Peterson 2003), but Bunces Pass had an unusually high spawning fraction of $97 \%$ (Lowerre-Barbieri et al. 2009). Due to the high spawning fraction, we made the assumption that individuals detected at the spawning site during the temporal window associated with spawning were participating in spawning. Therefore, site fidelity at this spawning aggregation indicates that individuals are leaving the site and returning to spawn at a later time. This temporal movement within the season may be driven by risk avoidance or foraging behaviors (Claydon et al. 2012, Becker \& Suthers 2014, Boucek et al. 2017). The results of inter-seasonal site fidelity over 3 spawning seasons were similar to those seen in 2005, when the spotted seatrout spawning season at Bunces was truncated due to a red tide (LowerreBarbieri et al. 2013).
This study is the first to assess inter-annual site fidelity of spotted seatrout at Bunces Pass, which was considerably lower than that demonstrated by common snook spawning at this same site during the same years (24 versus 50\%; Lowerre-Barbieri et al. 2014). A wide range of factors could have affected the inter-annual site fidelity, including high fishing and natural mortality (Nieland et al. 2002), shifts in annual spawning site selection, or skipped spawning (Rideout et al. 2005). However, at the population scale, a spotted seatrout spawning aggregation has been recorded at this site for more than 10 yr (S. Lowerre-Barbieri pers. obs.), indicating that individuals consistently select this area to spawn, even if the driver is unknown.

\subsection{Movement}

Due to the energetic requirements and increased predation risk associated with traveling large distances, fish are expected to minimize costs by participating in spawning aggregations that are nearby (Claydon et al. 2012). Yet other studies have found that factors such as social learning and female choice have a greater impact on spawning site selection than distance (Warner 1987). We found that some individuals traveled over $2 \mathrm{~km}$ within the array, passing other identified spotted seatrout spawning sites (Walters et al. 2009) to reach Bunces. Although we were only able to detect movements within the array, detections in the furthest east zone (zone 6) indicate fish moved to the spawning site from within Tampa Bay (70\% of the 43 tagged fish) while detections in the other zones suggest that individuals utilized the habitat near the spawning site. This suggests the Bunces Pass FSA draws fish from a larger area of the bay, and that fish undergo spawning migrations to this site. Spotted seatrout have traditionally been considered resident spawners that form aggregations within their home ranges and do not migrate to spawn (Walters et al. 2007). However, this is not the case at the Bunces FSA site (Lowerre-Barbieri et al. 2013, Boucek et al. 2017), which also falls outside of common spawning habitat. Similar behavior has been observed in coral trout, where populations form primary and secondary spawning aggregations (Samoilys 1997). Primary sites are those with the largest aggregations, but spawning is not limited to those sites and can occur at other secondary sites. In this case, Bunces Pass would be considered a primary site, with other secondary sites occurring throughout Tampa Bay. While this behavior has been observed in 
Tampa Bay, it is not yet known if spotted seatrout in other estuaries also may have primary and secondary aggregation sites.

We found sex-specific movements as described in other studies (Callihan et al. 2013) but not seen in network analysis based on detections in 2007 (Boucek et al. 2017). Significantly more females $(81.5 \% ; n=22)$ than males $(50 \% ; \mathrm{n}=8)$ were detected in the easternmost zone of the array, farthest from the spawning site (zone 6). Although there were no sex-specific differences in the total distance traveled over a spawning season, females exhibited larger maximum distances traveled within the array. Total distance will be impacted by sex-specific spawning frequency and loss rate, while maximum distance indicates how far an individual traveled between detections within the array, with females traveling greater distances during a single migration and males traveling shorter distances more frequently. These results indicate that females traveled large distances to participate in the aggregation at Bunces Pass while males might have been more opportunistic spawners, picking closer aggregations or remaining around the aggregation site. Similar patterns have been observed in cod, with females showing more fidelity to particular spawning sites than males (Dean et al. 2014). Understanding these sex-specific movements has important implications for estimating the true sex ratio of a population (Dean et al. 2014), as well as evaluating the effectiveness of spatial management or probability of capture.

\subsection{Spawning period}

Population spawning seasonality and individual spawning periods may be poorly correlated, with important implications for estimating TEP. The latest spotted seatrout stock assessment used a population spawning season of 184 d (Murphy et al. 2011), similar to what we saw based on the first and last detections at the FSA site (176 d in 2008; 159 d in 2009). However, individual spawning periods at this FSA site were quite variable and shorter, with a mean of $54.9 \mathrm{~d}$ and a range of 4-136 d. Although these estimations are solely for the FSA and do not account for spawning at other locations, if they are indicative of individual spawning periods they would significantly decrease TEP estimates. Support for our estimates to indicate spawning period comes from larger individuals exhibiting longer spawning periods, as this is similar to other studies which have reported larger, older females spawn for longer periods than younger females (DeMartini \& Fountain 1981, Wright \& Trippel 2009).

\subsection{Spawning interval}

Lowerre-Barbieri et al. (2011) estimated the spawning fraction at Bunces Pass to be $97 \%$ using capturebased methods. If spawning interval is estimated as the reciprocal of the spawning fraction (Hunter \& Macewicz 1985), the estimated interval for individuals at this FSA would be $1.03 \mathrm{~d}$ for females and unknown for males. Although our sample sizes were small, results from the CJS model with sex and TL bins support previous research that found older, larger females spawn more frequently than younger, new spawners (Lowerre-Barbieri et al. 2009, Fitzhugh et al. 2012, Porch et al. 2015). However, our spatially explicit individual spawning intervals indicated that although fish spawn at this site daily, individuals spawn here from once every $1.5-34 \mathrm{~d}$, with a population mean of $7.94 \mathrm{~d}$ for females and $3.06 \mathrm{~d}$ for males. These results are similar to spatially explicit estimates of individual spawning intervals conducted at this site in 2005 prior to a red tide causing the FSA to shut down mid-way through the season. In that study, female intervals were $9.3 \mathrm{~d}$ and male intervals were $2.2 \mathrm{~d}$ (Lowerre-Barbieri et al. 2013). Again, this has important implications for estimating TEP, especially spawning frequency. In fecundity studies, the assumption of no-movement to and from the spawning site is rarely tested - such as seen at this spawning site - and spawning frequency is assumed to be invariable over time. Of course, traditional methods also do not measure male spawning frequency, as it is not needed to estimate egg production. But the proper male to female sex ratio will be needed for the mating strategy of any given species, and can have serious impacts on fertilization rates and thus reproductive success.

\subsection{Apparent loss}

In this study, loss encompassed all ways that an individual may leave the spawning population (i.e. fishing mortality, natural mortality, tag battery death, and emigration). The model was not able to discern whether an individual died, finished spawning for the season, or left the spawning population to spawn elsewhere. We detected significant differences in apparent loss between sexes, but TL bins were not significant. If all loss was due to mortality, our estimates would correlate to a $Z$ of 2.55 for females and 5.3 for males. These are much higher than reported estimates of $Z$ for spotted seatrout in Tampa Bay $(0.77$ for females, 0.82 for males; Carroll \& Lowerre-Barbi- 
eri 2019). However, our estimated higher probability of loss from the population for males agrees with the reported increased natural mortality for males (Carroll \& Lowerre-Barbieri 2019). Thus, the loss rates for spawning at this site are much higher than expected from mortality and reflect a number of processes not typically considered in estimates of TEP, including individual spawning periods which are variable and much shorter than those of the spawning population (Wright \& Trippel 2009), potential shifts in spawning site selection within a spawning period, and potential for increased fishing and natural mortality when fish aggregate to spawn.

\subsection{Management implications}

By estimating spatially explicit, individual spawning frequencies, we have been able to demonstrate a range of factors not considered in traditional estimates of TEP as a measure of reproductive potential and highlight that traditional methods for estimating spawning frequency are inadequate if fish move to and from a spawning site. However, a short-coming of this study was an inability to test if individuals spawning at this FSA site also spawned elsewhere. Currently, we do not have the tagging technology to collect high-resolution tracks over small spatial scales, nor a movement signature that can be directly correlated with spawning activity. However, this is expected to change in the next decade (LowerreBarbieri et al. 2019a), and this study sets the foundation for developing more accurate estimates of spawning parameters needed for stock assessments to accurately assess the effects of age-truncation on spawning potential ratio estimates (Cooper et al. 2013).

Our results move beyond traditional measures of reproductive potential and begin the process of assessing how reproductive behavior may affect reproductive success (Lowerre-Barbieri et al. 2017). Unlike previous research that has indicated that spotted seatrout participate in spawning aggregations in their estuarine feeding ranges (Brown-Peterson 2003), Lowerre-Barbieri et al. (2013) found that individuals were traveling specifically to this site to spawn, and we were able to show that males and females exhibited not just significantly different spawning intervals but movement patterns within the array to this FSA site, suggesting males might be opportunistic spawners that participate in aggregations nearby while females are willing to expend more energy to travel to Bunces Pass.
Spawning site selection plays a role in reproductive success by influencing the environmental conditions that larvae and eggs encounter (Lowerre-Barbieri et al. 2017), and productive sites with favorable conditions for spawning can be source populations with higher productivity (Cadrin \& Secor 2009). If Bunces Pass is a productive spawning site with favorable conditions for spawning, it could be a source population with higher productivity than other estuarine sites. Such spawning 'hot spots' have been reported for other species (Carter et al. 2017, Ceriani et al. 2017), and spatial management of these areas could be beneficial for stock stability if they are heavily and successfully fished. However, to confirm this will necessitate determining if the Bunces FSA site disproportionately contributes to productivity in Tampa Bay.

\section{CONCLUSIONS}

In this study, we were able to demonstrate limitations of previously accepted methods to estimate spawning frequency, but we did not monitor additional seatrout FSA sites. Thus, we could not discern whether individuals spawning at Bunces might have also spawned at other locations, limiting our ability to make inferences about annual fecundity and population parameters. Also, our results demonstrated sizeand sex-specific spawning activity with important implications for other batch spawners with indeterminate fecundity. But like most telemetry studies, our conclusions were limited due to small sample sizes. Future studies should estimate a priori the sample size needed by sex and size bin to effectively test how these factors affect spawning frequency.

Estimates of individual spatially explicit spawning parameters are expected to become more common in the future, given our emerging understanding of how they affect estimates of TEP, a common measure of reproductive potential. However, for the methodology we present here to be applicable to other species and locations, a number of factors must be taken into consideration. First, because the spawning fraction at this site was previously estimated at $97 \%$, we were able to make the assumption that fish which moved to this site during the spawning season were spawning. However, many FSA sites have lower spawning fractions. Thus, site-specific probabilities of spawning when a fish is detected at an FSA site would need to be calculated. This is possible for females, given histological markers of spawning activity, but would not be possible for males. In the future this problem 
may be overcome based on 3D movement signatures indicating spawning events. Additionally, we assumed lack of detection meant fish were not at the spawning site. This assumption was possible given how we set up our array design: mapping the spawning site area based on courtship sound production and active spawners (Lowerre-Barbieri et al. 2013) and determining the minimum range bubble size for long-term detection at $50 \%$ or better to calculate the number of receivers needed to effectively monitor the FSA site. This detailed approach to range testing is becoming increasingly common, as is the use of sentinel tags to track array efficacy, as studies using virtual positioning systems become more common. In addition, acoustics are increasingly being used to help map and estimate the abundance at FSA sites (Lowerre-Barbieri et al. 2019b), and these approaches integrated with individual tracking are expected to change our understanding of spawnerrecruit systems.

Acknowledgements. We thank D. Westmark, J. Tunnell, J. Carroll, and A. Amick for their assistance in the field.

\section{LITERATURE CITED}

Becker A, Suthers IM (2014) Predator driven diel variation in abundance and behaviour of fish in deep and shallow habitats of an estuary. Estuar Coast Shelf Sci 144:82-88

Beverton RJH, Holt SJ (1957) On the dynamics of exploited fish populations. Ministry of Agriculture, Fisheries and Food, London

Boucek RE, Leone E, Bickford J, Walters-Burnsed S, Lowerre-Barbieri S (2017) More than just a spawning location: examining fine scale space use of two estuarine fish species at a spawning aggregation site. Front Mar Sci 4: 355

Brown-Peterson NJ (2003) The reproductive biology of spotted seatrout. In: Bortone S (ed) Biology of the spotted seatrout. CRC Press, Boca Raton, FL, p 99-133

Brown-Peterson NJ, Warren JW (2001) The reproductive biology of spotted seatrout, Cynoscion nebulosus, along the Mississippi Gulf coast. Gulf Mex Sci 2001:61-73

Cadrin SX, Secor DH (2009) Accounting for spatial population structure in stock assessment: past, present, and future. In: Beamish RJ, Rothschild BJ (eds) The future of fisheries science in North America. Fish \& Fisheries Series, Vol 31. Springer Science + Business Media, Dordrecht, p 405-426

* Calenge C (2006) The package adehabitat for the R software: a tool for the analysis of space and habitat use by animals. Ecol Model 197:516-519

Callihan JL, Cowan JH, Harbison MD (2013) Sex differences in residency of adult spotted seatrout in a Louisiana Estuary. Mar Coast Fish 5:79-92

Carroll J, Lowerre-Barbieri SK (2019) Interactions of dimorphic growth, reproductive behavior, and a size-regulated fishery: a case study using spotted seatrout Cynoscion nebulosus. Mar Ecol Prog Ser 608:233-245
Carter AB, Davies CR, Emslie MJ, Mapstone BD, Russ GR, Tobin AJ, Williams AJ (2017) Reproductive benefits of no-take marine reserves vary with region for an exploited coral reef fish. Sci Rep 7:9693

Ceriani SA, Weishampel JF, Ehrhart LM, Mansfield KL, Wunder MB (2017) Foraging and recruitment hotspot dynamics for the largest Atlantic loggerhead turtle rookery. Sci Rep 7:16894

Charnov E (1976) Optimal foraging, the marginal value theorem. Theor Popul Biol 9:129-136

Ciannelli L, Bailey K, Olsen EM (2015) Evolutionary and ecological constraints of fish spawning habitats. ICES J Mar Sci 72:285-296

Claydon JAB, McCormick MI, Jones GP (2012) Patterns of migration between feeding and spawning sites in a coral reef surgeonfish. Coral Reefs 31:77-87

* Cooper WT, Barbieri LR, Murphy MD, Lowerre-Barbieri SK (2013) Assessing stock reproductive potential in species with indeterminate fecundity: effects of age truncation and size-dependent reproductive timing. Fish Res 138: $31-41$

* Crossin GT, Heupel MR, Holbrook CM, Hussey NE and others (2017) Acoustic telemetry and fisheries management. Ecol Appl 27:1031-1049

* Dean MJ, Hoffman WS, Armstrong MP, Zemeckis DR (2014) Fine-scale diel and gender-based patterns in behavior of Atlantic cod (Gadus morhua) on a spawning ground in the western Gulf of Maine. ICES J Mar Sci 71:1474-1489

DeMartini EE, Fountain RK (1981) Ovarian cycling frequency and batch fecundity in the queenfish, Seriphus politus: attributes representative of serial spawning fishes. Fish Bull 79:547-560

*Dorazio RM (2016) Bayesian data analysis in population ecology: motivations, methods, and benefits. Popul Ecol $58: 31-44$

* Douglas SG, Chaput G, Hayward J, Sheasgreen J (2009) Prespawning, spawning, and postspawning behavior of striped bass in the Miramichi River. Trans Am Fish Soc 138:121-134

Espinoza M, Farrugia TJ, Webber DM, Smith F, Lowe CG (2011) Testing a new acoustic telemetry technique to quantify long-term, fine-scale movements of aquatic animals. Fish Res 108:364-371

Fitzhugh GR, Shertzer KW, Kellison GT, Wyanski DM (2012) Review of size- and age-dependence in batch spawning: implications for stock assessment of fish species exhibiting indeterminate fecundity. Fish Bull 110:413-425

Hilbe J, Robinson A (2018) msme: functions and datasets for methods of statistical model estimation. $\mathrm{R}$ package version 0.5.3. https://CRAN.R-project.org/package=msme

Hunter JR, Macewicz BJ (1985) Measurement of spawning frequency in multiple spawning fishes. In Lasker R (ed) An egg production method for estimating spawning biomass of pelagic fishes: application to the northern anchovy, Engraulis mordax. NOAA Tech Rep NMFS 36: 79-94

Kéry M, Schaub M (2012) Bayesian population analysis using WinBUGS. A hierarchical perspective. Academic Press, Waltham, MA

* Lambert TC (1987) Duration and intensity of spawning in herring Clupea harengus as related to the age structure of the mature population. Mar Ecol Prog Ser 39:209-220

* Lowerre-Barbieri SK, Henderson N, Llopiz J, Walters S, Bickford J, Muller R (2009) Defining a spawning population of spotted seatrout (Cynoscion nebulosus) over tem- 
poral, spatial, and demographic scales. Mar Ecol Prog Ser 394:231-245

Lowerre-Barbieri SK, Ganias K, Saborido-Rey F, Murua H, Hunter JR (2011) Reproductive timing in marine fishes: variability, temporal scales, and methods. Mar Coast Fish 3:71-91

Lowerre-Barbieri SK, Walters SL, Bickford JW, Cooper W, Muller R (2013) Site fidelity and reproductive timing at a spotted seatrout spawning aggregation site: individual versus population scale behavior. Mar Ecol Prog Ser 481: 181-197

Lowerre-Barbieri SK, Villegas-Ríos D, Walters S, Bickford J, Cooper W, Muller R, Trotter A (2014) Spawning site selection and contingent behavior in common snook, Centropomus undecimalis. PLOS ONE 9:e101809

Lowerre-Barbieri SK, Walters Burnsed SL, Bickford JW (2016) Assessing reproductive behavior important to fisheries management: a case study with red drum, Sciaenops ocellatus. Ecol Appl 26:979-995

Lowerre-Barbieri S, DeCelles G, Pepin P, Catalán IA and others (2017) Reproductive resilience: a paradigm shift in understanding spawner-recruit systems in exploited marine fish. Fish Fish 18:285-312

* Lowerre-Barbieri SK, Kays R, Thorson JT, Wikelski M (2019a) The ocean's movescape: fisheries management in the bio-logging decade (2018-2028). ICES J Mar Sci 76:477-488

Lowerre-Barbieri SK, Tringali MD, Shea CP, Walter Burnsed S, Bickford J, Murphy M, Porch C (2019b) Assessing red drum spawning aggregations and abundance in the eastern Gulf of Mexico: a multidisciplinary approach. ICES J Mar Sci 76:516-529

'Marshall TC, O'Brien L, Tomkiewicz J, Köster FW and others (2003) Developing alternative indices of reproductive potential for use in fisheries management: case studies for stocks spanning an information gradient. J Northwest Atl Fish Sci 33:161-190

Marshall TC, Kjesbu OS, Yaragina NA, Solemdal P, Ulltang O (1998) Is spawning biomass a sensitive measure of the reproductive and recruitment potential of Northeast Artic cod? Can J Fish Aquat Sci 55:1766-1783

Murphy MD, Chagaris D, Addis D (2011) An assessment of the status of spotted seatrout in Florida waters through 2009. Report IHR 2011-2. Florida Fish and Wildlife Conservation Commission, Fish and Wildlife Research Institute, St. Petersburg, FL

Murua H, Saborido-Rey F (2003) Female reproductive strategies of marine fish species of the North Atlantic. J Northwest Atl Fish Sci 33:23-31

Nieland DL, Thomas RG, Wilson CA (2002) Age, growth, and reproduction of spotted seatrout in Barataria Bay, Louisiana. Trans Am Fish Soc 131:245-259

Plummer M (2016) rjags: Bayesian graphical models using MCMC. R package version 4-6. https://CRAN.R-project. org/package=rjags

Plummer M, Best N, Cowles K, Vines K (2006) CODA: convergence diagnosis and output analysis for MCMC. R News 6:7-11

Porch CE, Fitzhugh GR, Lang ET, Lyon HM, Linton BC (2015) Estimating the dependence of spawning frequency on size and age in Gulf of Mexico red snapper.
Mar Coast Fish 7:233-245

R Core Team (2017) R: a language and environment for statistical computing. R Foundation for Statistical Computing, Vienna

Ricker WE (1954) Stock and recruitment. J Fish Res Board Can 11:559-623

Rideout RM, Rose GA, Burton MPM (2005) Skipped spawning in female iteroparous fishes. Fish Fish 6:50-72

* Robichaud D, Rose GA (2003) Sex differences in cod residency on a spawning ground. Fish Res 60:33-43

Rothschild BJ, Fogarty MJ (1989) Spawning-stock biomass: a source of error in recruitment/stock relationships and management advice. ICES J Mar Sci 45:131-135

Russell MW, Luckhurst BE, Lindeman KC (2012) Management of spawning aggregations. In: de Mitcheson YS, Colin PL (eds) Reef fish spawning aggregations: biology, research and management. Springer, New York, NY, p 371-404

Samoilys MA (1997) Periodicity of spawning aggregations of coral trout Plectropomus leopardus (Pisces: Serranidae) on the northern Great Barrier Reef. Mar Ecol Prog Ser 160:149-159

Secor DH (2000) Spawning in the nick of time? Effect of adult demographics on spawning behavior and recruitment in Chesapeake Bay striped bass. ICES J Mar Sci 57: 403-411

* Semmens J, Buxton C, Forbes E, Phelan M (2010) Spatial and temporal use of spawning aggregation sites by the tropical sciaenid Protonibea diacanthus. Mar Ecol Prog Ser 403:193-203

พ Siceloff L, Howell WH (2013) Fine-scale temporal and spatial distributions of Atlantic cod (Gadus morhua) on a western Gulf of Maine spawning ground. Fish Res 141:31-43

Simpfendorfer CA, Heupel MR, Hueter RE (2002) Estimation of short-term centers of activity from an array of omnidirectional hydrophones and its use in studying animal movements. Can J Fish Aquat Sci 59:23-32

Stearns SC (1992) The evolution of life histories. Oxford University Press, Oxford

* Trippel EA (1999) Estimation of stock reproductive potential: history and challenges for Canadian Atlantic gadoid stock assessments. J Northwest Atl Fish Soc 25:61-81

Walters S, Lowerre-Barbieri S, Bickford J, Crabtree L, Mann D (2007) Preliminary results on seasonal and diel periodicities of a resident Cynoscion nebulosus spawning aggregation in Tampa Bay, Florida. Gulf Caribb Fish Inst 58:281-285

Walters S, Lowerre-Barbieri S, Bickford J, Mann D (2009) Using a passive acoustic survey to identify spotted seatrout spawning sites and associated habitat in Tampa Bay, Florida. Trans Am Fish Soc 138:88-98

*Warner RR (1987) Female choice of sites versus mates in coral reef fish, Thalassoma bifasciatum. Anim Behav 35: 1470-1478

* Wright PJ, Trippel EA (2009) Fishery-induced demographic changes in the timing of spawning: consequences for reproductive success. Fish Fish 10:283-304

*Young JM, Yeiser BG, Ault ER, Whittington JA, DutkaGianelli J (2016) Spawning site fidelity, catchment, and dispersal of common snook along the east coast of Florida. Trans Am Fish Soc 145:400-415

Submitted: June 20, 2018; Accepted: May 31, 2019

Proofs received from author(s): July 25, 2019
Editorial responsibility: Stylianos Somarakis, Heraklion, Greece 